Article

\title{
Homoleptic Lanthanide Complexes Containing a Redox-Active Ligand and the Investigation of Their Electronic and Photophysical Properties
}

\author{
Robin A. Taylor ${ }^{1,2}$, Nico M. Bonanno ${ }^{2}$, Mihaela Cibian ${ }^{3}$, Jeetika Yadav ${ }^{3}$ (D), \\ Harlyn J. Silverstein ${ }^{1}$, Christopher R. Wiebe ${ }^{1,4,5}$, Cole Mauws ${ }^{1}$ (D), Alan J. Lough ${ }^{6}$ \\ and Martin T. Lemaire $1,2,7, *$ (D) \\ 1 Department of Chemistry, University of Manitoba, Winnipeg, MB R3T 2N2, Canada; \\ rob2235@hotmail.com (R.A.T.); harlyn.silverstein@gmail.com (H.J.S.); chris.r.wiebe@gmail.com (C.R.W.); \\ colemws@gmail.com (C.M.) \\ 2 Department of Chemistry, Brock University, St. Catharines, ON L2S 3A1, Canada; nb15sz@brocku.ca \\ 3 Department of Chemistry, Université de Montreal, Montreal, QC H3C 3J7, Canada; \\ cibian.mihaela@gmail.com (M.C.); jeetika.yadav@gmail.com (J.Y.) \\ 4 Department of Chemistry, University of Winnipeg, Winnipeg, MB R3B 2G3, Canada \\ 5 Canadian Institute for Advanced Research, Quantum Materials, MaRS Centre, West Tower, \\ 661 University Ave., Suite 505, Toronto, ON M5G 1M1, Canada \\ 6 Department of Chemistry, University of Toronto, 80 St. George Street, Toronto, ON M5S 3H6, Canada; \\ alough@chem.utoronto.ca \\ 7 Department of Chemistry, Brandon University, Brandon, MB R7A 6A9, Canada \\ * Correspondence: mlemaire@brocku.ca; Tel.: +1-905-688-5550
}

Received: 19 March 2018; Accepted: 18 May 2018; Published: 25 May 2018

\begin{abstract}
Herein, we describe the preparation, characterization and photophysical properties of neutral lanthanide complexes containing a redox-active ligand 1-(2-pyridylazo)-2-phenanthrol (papl). The complexes likely share similar structural features and bear the formulation $\operatorname{Ln}(\mathbf{p a p l})_{3}$ $(\mathrm{Ln}(\mathrm{III})=\mathrm{Gd}, \mathrm{Dy}, \mathrm{Tb})$, which is supported by electrospray ionization mass spectrometry, CHN analysis, FT-IR and UV-Vis spectroscopy. The synthesis and structural properties of a related complex, $\mathrm{Ho}(\mathbf{q a p l})_{3}$ (where qapl = 10-(8-quinolylazo)-9-phenanthrol), is also reported. The complexes feature ligand-centered redox activity, similar to other reported transition metal complexes with papl. Variable temperature magnetic susceptibility measurements (DC and AC) suggest typical free-ion magnetism without any slow-relaxation dynamics. The photophysical properties of the ligand and complexes were investigated and the results of emission spectroscopy indicate ligand-centered processes.
\end{abstract}

Keywords: redox active ligands; arylazo ligands; lanthanide ions; photophysical properties

\section{Introduction}

Lanthanide ion complexes are of interest for the production of luminescent and magnetic materials, including as diagnostic agents in magnetic resonance imaging (MRI) [1-3], biosensors [4,5], and single molecule (or ion) magnets (SMMs and SIMs, respectively) [6,7]. The contracted nature of the $4 f^{n}$ valence orbitals of lanthanide ions results in little to no perturbation of the electronic structure within the lanthanide ion upon ligand binding. Very often, the observed luminescence in these complexes is the result of transitions within the energy level manifold of the lanthanide ion and this generally results in the characteristic sharp emission spectra observed for these complexes. Another result of the isolation of the $f$-orbitals from the ligand field is that lanthanide ion complexes often exhibit unique 
magnetic properties generally easy to interpret as free ion magnetism by consideration of the total angular momentum of the ion in the form of the free ion term symbol. Depending on the energy level structure and depopulation of higher lying $J$ states, a temperature dependence of the magnetic moment is observed. Under the right set of conditions that include symmetry, coordination number, geometry and ligand field electrostatics, strong magnetoanisotropy is observed resulting in barriers to magnetization relaxation and the observation of single ion magnetism [8].

We have been interested in the coordination chemistry of redox-active ligands, including those in the arylazo family [9]. Arylazo ligands feature low-lying $\pi^{*}$ azo-centered molecular orbitals and often exhibit intense visible absorption bands resulting from electronic transitions into these orbitals. The tridentate 1-(2-pyridylazo)-2-phenanthrol (papl) ligand has been investigated since the 1960s [10] and we have recently taken another look at the coordination chemistry of this structurally diverse ligand and other derivatives. The papl structure (Chart 1) contains pyridyl, azo, and phenanthrol fragments and is susceptible to either reduction or oxidation. Of note, first row transition metal complexes containing papl exhibit very intense ligand-centered absorption bands in the visible region, reversible cathodic electrochemistry and we have recently observed thermally induced spin-crossover in $\mathrm{Co}(\mathbf{p a p l})_{2}$ [11,12]. Iron complexes containing other arylazo substituted ligands have also recently been shown to exhibit spin-crossover and there is the potential to create multifunctional materials using these ligands with interesting magnetic, optical and electronic properties [13]. To date, there are no reported lanthanide ion complexes containing papl. However, given the interest in both redox-active ligands and lanthanide ion complexes, we believe the coordination chemistry of papl with lanthanide ions potentially has much to offer multifunctional materials research. Herein, we present the preparation and characterization of three lanthanide complexes containing papl in an effort to unearth the details regarding the electronic structures and photophysical properties in these interesting complexes. We also report on the structural properties of a holmium complex with the related ligand qapl. To our knowledge, this is the first report focused on the emission properties of homoleptic lanthanide complexes containing arylazo ligands and also the first reported lanthanide complexes with papl.<smiles>Oc1c(/N=N/c2ccccn2)c2ccccc2c2ccccc12</smiles><smiles>Oc1c(/N=N/c2cccc3cccnc23)c2ccccc2c2ccccc12</smiles>

qapl

Chart 1. Arylazo ligands used in the present study.

\section{Results and Discussion}

\subsection{Preparation and Characterization of $\mathrm{Ln}(\mathbf{p a p l})_{3} \mathrm{Ln}=\mathrm{Gd}, \mathrm{Tb}, \mathrm{Dy}$ and $\mathrm{Ho}(\mathrm{qapl})_{3}$ Complexes}

The $\operatorname{Ln}(\mathbf{p a p} 1)_{3}$ complexes were easily prepared by combination of the appropriate amount of lanthanide nitrate salt in methanol with a chloroform solution containing three equivalents of papl and triethylamine (Scheme 1). Stirring at room temperature precipitated out dark microcrystalline materials that are air/moisture stable. Unfortunately, multiple attempts to grow single crystals of the complexes for X-ray diffraction experiments were unsuccessful. Often, microcrystalline clusters or microspherical particles were formed by slow evaporation or diffusion experiments. However, it is clear from the results of combustion analysis and ESI mass spectrometry that complexes 1-3 bear the formulation $\operatorname{Ln}(\mathbf{p a p} \mathbf{1})_{3}$. Dominant peaks are observed by ESI (Figures S1-S6) in all cases at high $\mathrm{m} / \mathrm{z}$ that represent $\left[\mathrm{Ln}(\mathbf{p a p l})_{3} \mathrm{H}\right]^{+}$with isotope distributions that match the theoretical calculated isotopic 
patterns; combustion analysis results are also consistent with this formulation (with the addition of chloroform for $\mathrm{Tb}^{3+}$ complex only). The FT-IR and UV-Vis spectra of 1-3 are identical, and similar electrochemical data are observed (vide infra), strongly suggesting that 1-3 share similar structural properties. It is likely that the molecular structure of complexes 1-3 consist of nine-coordinate $\mathrm{Ln}^{3+}$ ions in tricapped trigonal prismatic geometries, which have been observed previously for other tridentate NNO ligands, where each papl ligand is binding the lanthanide ion through pyridyl N, azo $\mathrm{N}$ and anionic phenanthrol $\mathrm{O}$ atoms [14]. Using a related ligand, qapl (Chart 1) [15], we prepared an analogous holmium complex $\mathrm{Ho}(\mathbf{q a p l})_{3} 4$, which provided spectroscopic data that was similar to 1-3, including an ESI mass spectrum that indicated $m / z$ corresponding to $\left[\mathrm{Ho}(\mathbf{q a p l})_{3} \mathrm{H}\right]^{+}$. Single crystals of 4 were obtained by slow evaporation of $\mathrm{DCM} / \mathrm{CH}_{3} \mathrm{CN}$ solutions of the precipitated powder. The molecular structure of 4 is shown in Figure 1 (crystallographic parameters can be found in Table S1). There is substantial disorder in the structure that was modeled using geometric constraints. One of the ligands is disordered over the twofold crystallographic axis and is essentially superimposed upon itself. While $R_{1}$ is a little high (0.1087), we are confident in the overall atom connectivity and the coordinate bond features in the ordered parts of the molecule. The structural data confirms the nine-coordinate structure we suggested for $\operatorname{Ln}(\text { papl })_{3}$ complexes and given the spectroscopic similarities between complexes 1-4 it is likely that the structures of complexes 1-3 are similar to 4 . The coordinate bonds in 4 are shortest to the $\mathrm{O}$ donor atoms and longer to the $\mathrm{N}_{\text {azo }}$ and $\mathrm{N}_{\text {quin }}$ donors (Figure 1). The bond distances in the ligand are similar to those in $\left[\mathrm{Co}(\mathbf{q a p l})_{2}\right] \mathrm{Cl}$ reported earlier and indicate a bound qapl phenolate anion [15].

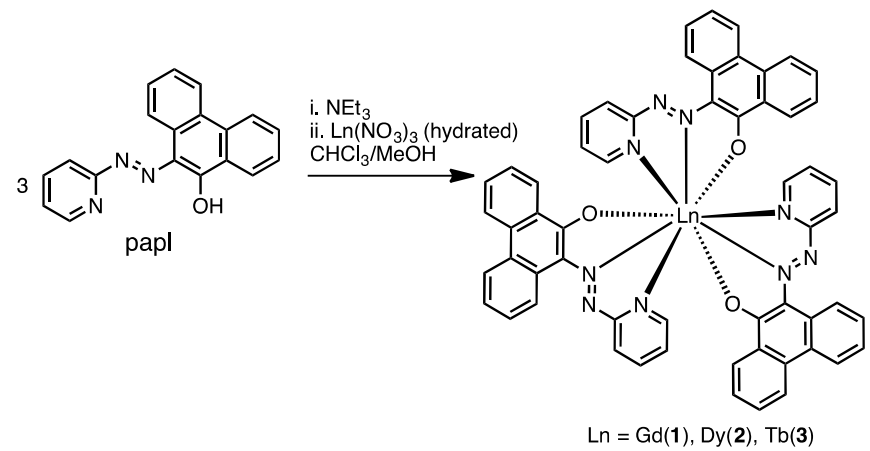

Scheme 1. Preparation of $\mathrm{Ln}(\text { papl })_{3}$ complexes. $\mathrm{Ho}(\text { qapl })_{3}(4)$ was prepared analogously using $\mathrm{HoCl}_{3}$ hexahydrate and qapl (Chart 1$)$.

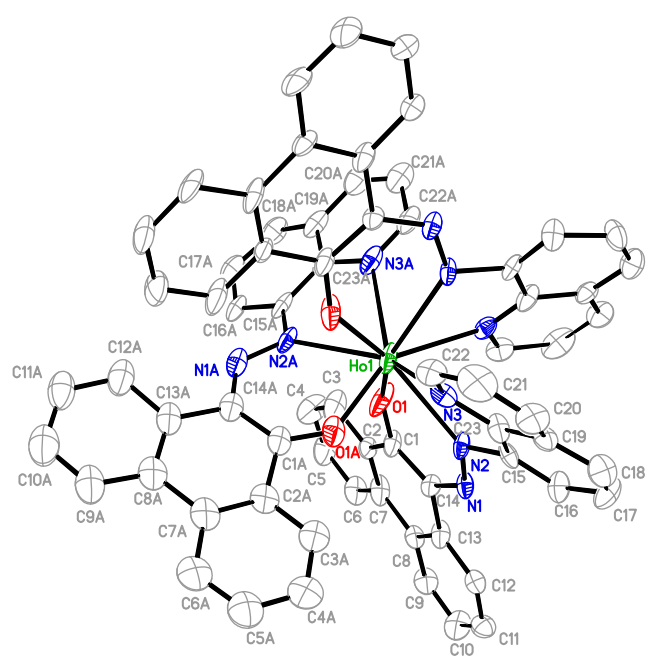

Figure 1. Molecular structure of 4 (displacement ellipsoids at $30 \%$ probability). $\mathrm{H}$ atoms removed for clarity. Complex 4 lies on a crystallographic twofold rotation axis and one of the qapl ligands is disordered 
about a twofold rotation axis over two sites with equal occupancy. In Figure 1, only one site is shown for the disordered ligand and the atom labels are denoted with suffix " $\mathrm{A}$ ". Unlabeled atoms in the figure and those in list below which are denoted by suffix "\#" are related by the symmetry operator $(-x+1, y,-z+3 / 2)$. Relevant bond distances $(\AA)$ and angles $\left(^{\circ}\right)$ [with standard uncertainties (su) in brackets]: $\mathrm{Ho}(1)-\mathrm{O}(1 \mathrm{~A}), 2.122(11) ; \mathrm{Ho}(1)-\mathrm{O}(1) \# 1,2.273(6) ; \mathrm{H}(1)-\mathrm{O}(1), 2.273(6) ; \mathrm{Ho}(1)-\mathrm{N}(2 \mathrm{~A})$, 2.555(10); $\mathrm{Ho}(1)-\mathrm{N}(2) \# 1,2.557(7) ; \quad \mathrm{Ho}(1)-\mathrm{N}(2), 2.557(7) ; \mathrm{Ho}(1)-\mathrm{N}(3) \# 1,2.596(8) ; \mathrm{Ho}(1)-\mathrm{N}(3)$, 2.596(8); $\mathrm{Ho}(1)-\mathrm{N}(3 \mathrm{~A}), 2.737(12) ; \mathrm{N}(1)-\mathrm{N}(2), 1.308(8) ; \mathrm{C}(1)-\mathrm{O}(1), 1.283(10) ; \mathrm{N}(1 \mathrm{~A})-\mathrm{N}(2 \mathrm{~A}), 1.303(11)$; $\mathrm{C}(1 \mathrm{~A})-\mathrm{O}(1 \mathrm{~A}), 1.276(12) . \mathrm{O}(1 \mathrm{~A})-\mathrm{Ho}(1)-\mathrm{O}(1) \# 1,84.3(4) ; \mathrm{O}(1 \mathrm{~A})-\mathrm{Ho}(1)-\mathrm{O}(1), 78.1(4) ; \mathrm{O}(1) \# 1-\mathrm{Ho}(1)-\mathrm{O}(1)$, 142.8(3); $\mathrm{O}(1 \mathrm{~A})-\mathrm{Ho}(1)-\mathrm{N}(2 \mathrm{~A}), 74.9(4) ; \mathrm{O}(1)-\mathrm{Ho}(1)-\mathrm{N}(2 \mathrm{~A}), 68.6(4) ; \mathrm{O}(1 \mathrm{~A})-\mathrm{Ho}(1)-\mathrm{N}(2) \# 1,143.5(4)$; $\mathrm{O}(1) \# 1-\mathrm{Ho}(1)-\mathrm{N}(2) \# 1, \quad 68.8(2) ; \quad \mathrm{O}(1)-\mathrm{Ho}(1)-\mathrm{N}(2) \# 1, \quad 137.8(3) ; \quad \mathrm{N}(2 \mathrm{~A})-\mathrm{Ho}(1)-\mathrm{N}(2) \# 1, \quad 121.2(4) ;$ $\mathrm{O}(1 \mathrm{~A})-\mathrm{Ho}(1)-\mathrm{N}(2), 76.6(4) ; \mathrm{O}(1) \# 1-\mathrm{Ho}(1)-\mathrm{N}(2), 137.8(3) ; \mathrm{O}(1)-\mathrm{Ho}(1)-\mathrm{N}(2), 68.8(2) ; \mathrm{N}(2 \mathrm{~A})-\mathrm{Ho}(1)-\mathrm{N}(2)$, 130.6(4); $\mathrm{N}(2) \# 1-\mathrm{Ho}(1)-\mathrm{N}(2), \quad 106.9(4) ; \quad \mathrm{O}(1 \mathrm{~A})-\mathrm{Ho}(1)-\mathrm{N}(3) \# 1, \quad 142.5(4) ; \quad \mathrm{O}(1) \# 1-\mathrm{Ho}(1)-\mathrm{N}(3) \# 1$, 131.2(2); $\quad \mathrm{O}(1)-\mathrm{Ho}(1)-\mathrm{N}(3) \# 1, \quad 77.3(3) ; \quad \mathrm{N}(2 \mathrm{~A})-\mathrm{Ho}(1)-\mathrm{N}(3) \# 1, \quad 123.4 ; \quad \mathrm{N}(2) \# 1-\mathrm{Ho}(1)-\mathrm{N}(3) \# 1$, 63.3(2); $\mathrm{N}(2)-\mathrm{Ho}(1)-\mathrm{N}(3) \# 1, \quad 68.3(2) ; \quad \mathrm{O}(1 \mathrm{~A})-\mathrm{Ho}(1)-\mathrm{N}(3), \quad 82.4(4) ; \quad \mathrm{O}(1) \# 1-\mathrm{Ho} 91)-\mathrm{N}(3), \quad 77.3(3)$; $\mathrm{O}(1)-\mathrm{Ho}(1)-\mathrm{N}(3), 131.2(2) ; \mathrm{N}(2 \mathrm{~A})-\mathrm{Ho}(1)-\mathrm{N}(3), 143.2(3) ; \mathrm{N}(2) \# 1-\mathrm{Ho}(1)-\mathrm{N}(3), 68.3(2) ; \mathrm{N}(2)-\mathrm{Ho}(1)-\mathrm{N}(3)$, 63.2(2); $\quad \mathrm{N}(3) \# 1-\mathrm{Ho}(1)-\mathrm{N}(3), \quad 93.1(3) ; \quad \mathrm{O}(1 \mathrm{~A})-\mathrm{Ho}(1)-\mathrm{N}(3 \mathrm{~A}), \quad$ 131.6(4); $\mathrm{O}(1) \# 1-\mathrm{Ho}(1)-\mathrm{N}(3 \mathrm{~A})$, 89.8(5); $\mathrm{O}(1)-\mathrm{Ho}(1)-\mathrm{N}(3 \mathrm{~A}), 78.4(4) ; \mathrm{N}(2 \mathrm{~A})-\mathrm{Ho}(1)-\mathrm{N}(3 \mathrm{~A}), 60.8(3) ; \mathrm{N}(2) \# 1-\mathrm{Ho}(1)-\mathrm{N}(3 \mathrm{~A}), 74.3(4)$; $\mathrm{N}(2)-\mathrm{Ho}(1)-\mathrm{N}(3 \mathrm{~A}), 130.7(4) ; \mathrm{N}(3) \# 1-\mathrm{Ho}(1)-\mathrm{N}(3 \mathrm{~A})$, 69.3(4); N(3)-Ho(1)-N(3A), 142.6(4).

The electronic spectra of the uncoordinated papl ligand and complexes 1-3 are illustrated in Figure 2. For the uncoordinated papl ligand, a keto-enol tautomerization exists (Scheme 2), with the iminoquinone tautomer being the major component, as found for related compounds [16]. In its absorption spectrum, ligand centered $\pi-\pi^{*}$ transitions $(225-350 \mathrm{~nm})$ are observed, together with a broad band (350-525 $\mathrm{nm}$ ) with a higher energy shoulder $(\sim 400 \mathrm{~nm})$ in the visible region. The latter feature is assigned to the $n-\pi^{*}$ transitions of the azo moiety [11]. The three $\operatorname{Ln}(\text { papl })_{3}$ complexes present nearly identical UV-vis spectra, which feature ligand centered $\pi-\pi^{*}$ transitions $(225-360 \mathrm{~nm})$, and two overlapping broad bands with a higher energy shoulder $(375-600 \mathrm{~nm})$ in the visible region, assigned as intraligand charge transfer transitions (ILCT) [11,17]. The red shift observed for the transitions in the visible region upon the complexation of the lanthanide ions is in line with what has been reported for other papl complexes with divalent transition metal ions [11], as well as for lanthanide complexes with similar azo-type ligands [18]. This effect arises upon coordination, as only the enolate form of the ligand with the azo-type moiety exists with extended conjugation and a characteristic low lying azo-centered $\pi^{*}$-orbital, which is also supported by the intense color of the complexes $[9,11,16,18]$.

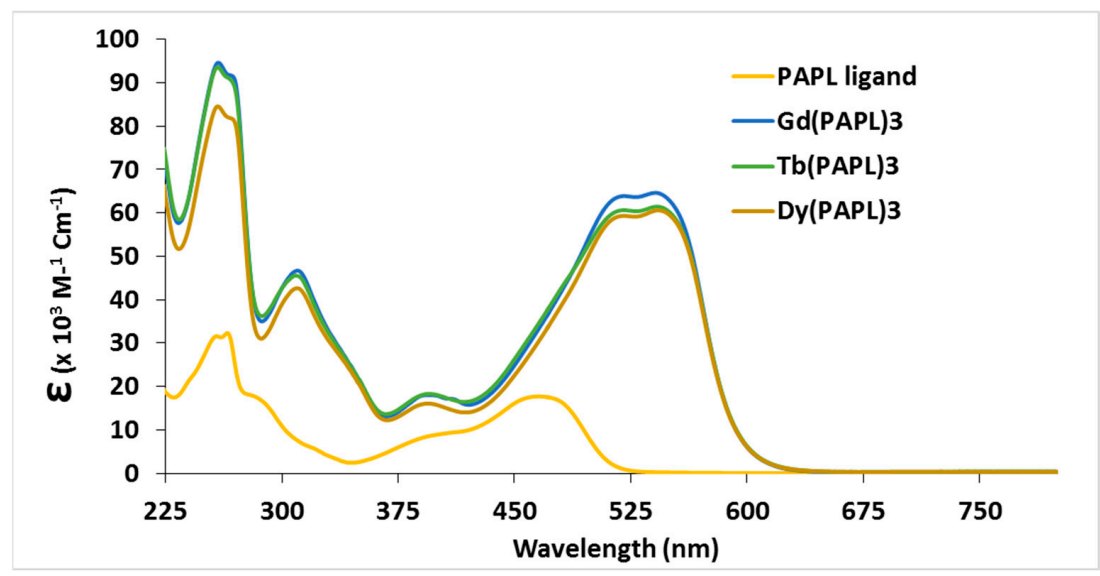

Figure 2. Electronic spectra of papl, $\mathrm{Gd}(\text { papl })_{3}(\mathbf{1}), \mathrm{Dy}(\text { papl })_{3}(2), \mathrm{Tb}(\text { papl })_{3}$ (3) in $\mathrm{CH}_{2} \mathrm{Cl}_{2}$ at room temperature. 
<smiles></smiles>

enol form<smiles></smiles>

keto form/ iminoquinone form

Scheme 2. Keto-enol tautomerization for the papl ligand showing intramolecular proton transfer.

\subsection{Electrochemical and Variable Temperature Magnetic Susceptibility Data}

We have previously described the redox-activity of papl, in the uncoordinated form and when coordinated to transition metal ions [11]. The anticipated redox behavior of papl is summarized in Scheme 3. The electrochemical properties of 1-3 were investigated by CV and DPV and the results are shown in Figures 3-5 for $\mathbf{1}$ and all data for $\mathbf{1}-\mathbf{3}$ is summarized in Table 1 (cyclic and differential pulse voltammograms for $\mathbf{2}$ and $\mathbf{3}$ are provided in Figures S7-S12). All three complexes share very similar electrochemical properties, which result from ligand-centered redox processes. Over anodic potentials, irreversible waves are found at high potentials $(>+0.9 \mathrm{~V}$, vs. $\mathrm{Ag} / \mathrm{AgCl})$, likely resulting from papl oxidation, as observed in related $\mathrm{M}(\mathbf{p a p l})_{2}$ complexes [11]. Two quasi-reversible cathodic waves are observed at potentials beyond $-1.0 \mathrm{~V}$ (vs. $\mathrm{Ag} / \mathrm{AgCl}$ ) for each of the complexes, which are assigned to ligand centered reduction processes. Complexes $\mathbf{1}$ and $\mathbf{3}$ exhibit a third irreversible process at very negative potentials $(>-1.8 \mathrm{~V})$ that is only observed in the differential pulse voltammogram. In $\mathrm{M}(\mathbf{p a p l})_{2}$ complexes the frontier molecular orbital structure is nearly exclusively papl in character, and similar behavior is anticipated for $\mathbf{1 - 3}$.

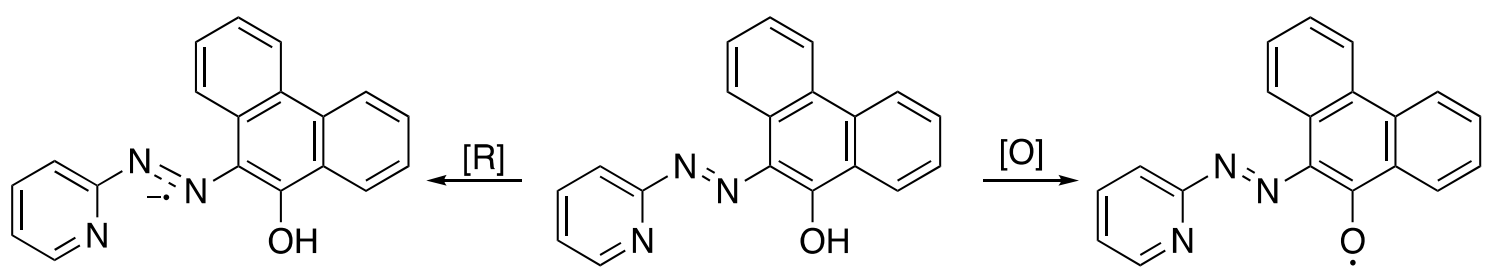

Scheme 3. Potential redox-activity anticipated for papl.

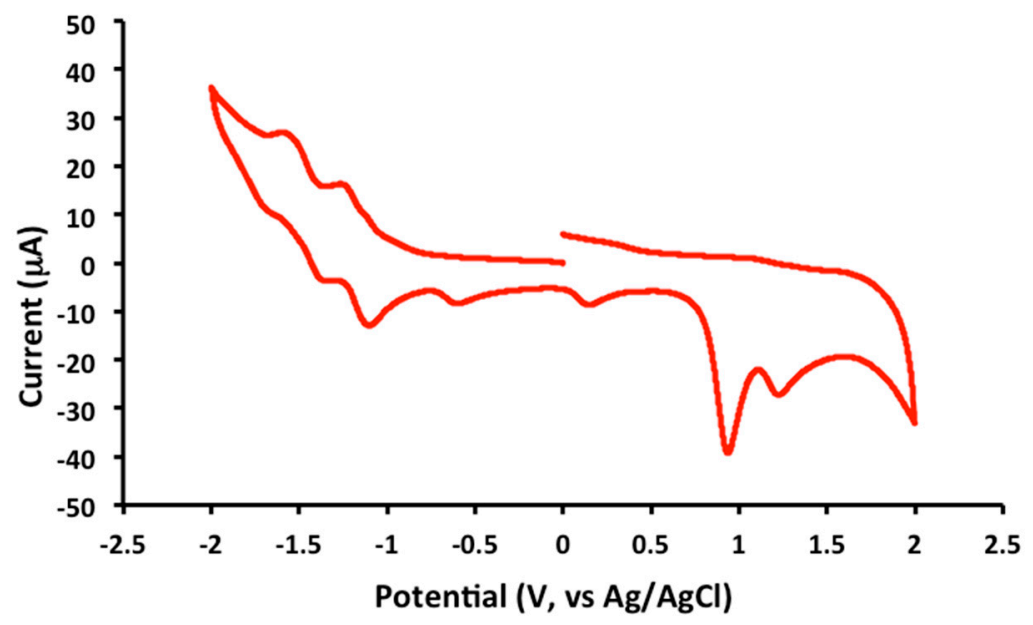

Figure 3. $\mathrm{CV}$ of 1 in $\mathrm{CH}_{2} \mathrm{Cl}_{2}$ (containing ca. $0.5 \mathrm{M} \mathrm{Bu}_{4} \mathrm{NPF}_{6}$; scan rate $200 \mathrm{mV} / \mathrm{s}$ ). 


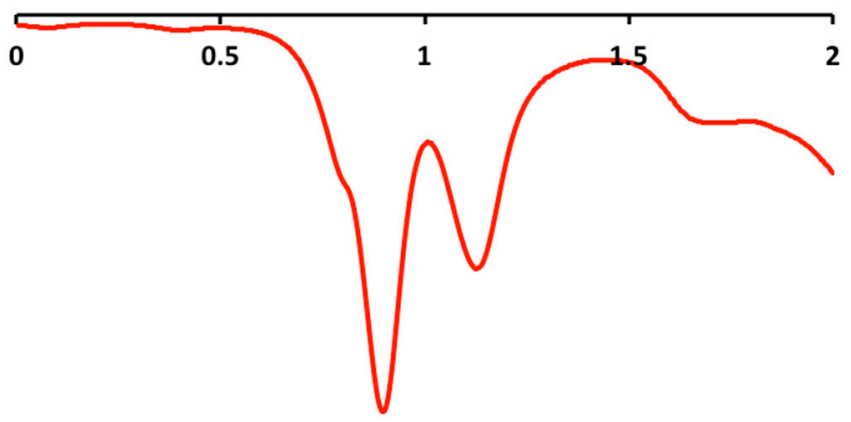

Potential (V vs Ag/AgCl)

Figure 4. Anodic DPV of 1 in $\mathrm{CH}_{2} \mathrm{Cl}_{2}$ with a scan rate of $50 \mathrm{mV} / \mathrm{s}$.

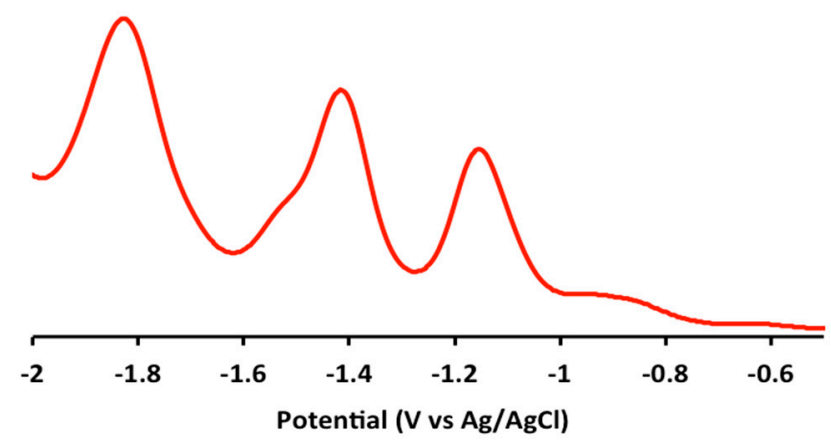

Figure 5. Cathodic DPV of $\mathbf{1}$ in $\mathrm{CH}_{2} \mathrm{Cl}_{2}$ with a scan rate of $50 \mathrm{mV} / \mathrm{s}$.

Table 1. Electrochemical properties of 1-3 (vs. Ag/ $\mathrm{AgCl}$ ).

\begin{tabular}{ccc}
\hline Compound & $E_{1 / 2}$ (Anodic) & $E_{1 / 2}$ (Cathodic) \\
\hline $\mathbf{1}$ & $1.2(\mathrm{irr}), 0.9$ (irr) & $-1.2(\mathrm{qr}),-1.5(\mathrm{qr}),-1.8$ (irr) \\
$\mathbf{2}$ & 1.2 (irr), 0.9 (irr) & $-1.2(\mathrm{qr}),-1.4(\mathrm{qr})$ \\
$\mathbf{3}$ & 1.2 (irr), $1.0(\mathrm{irr})$ & $-1.2(\mathrm{qr}),-1.5(\mathrm{qr}),-1.9$ (irr) \\
\hline
\end{tabular}

Variable temperature magnetic susceptibility experiments were conducted for all three complexes over a temperature range of 300-2 K at an external field of 2000 Oe on a Quantum Design PPMS (vibrating sample magnetometer option) for $\mathbf{1}$ and on a Quantum Design MPMS squid magnetometer for $\mathbf{2}$ and 3. The data obtained from samples of 1-3 (Figure 6) support the formulation as $\operatorname{Ln}(\mathbf{p a p l})_{3}$, with free-ion type magnetism characteristic of the $\mathrm{Gd}(\mathrm{III})$, Dy(III), or $\mathrm{Tb}(\mathrm{III})$ terms $\left({ }^{8} \mathrm{~S}_{7 / 2},{ }^{6} \mathrm{H}_{15 / 2}\right.$, or ${ }^{7} \mathrm{~F}_{6}$ ) exhibiting typical $\chi_{m} T$ values at $300 \mathrm{~K}$ of $7.6,13.3$, and $10.0 \mathrm{~cm}^{3} \mathrm{~K} \mathrm{~mol}^{-1}$ for $1-3$, respectively. At low temperatures, there are observed minor deviations from the above $\chi_{m} T$ values, primarily 2 and 3, indicating depopulation of Stark sublevels at these lower temperatures. Magnetization versus field experiments (Figure 7) at low temperature (2K) were also performed on all three complexes. Preliminary AC SQUID experiments indicated no out-of-phase peaks at zero external field or in an external DC field; therefore, 1-3 do not exhibit slow relaxation of the magnetization, which is a characteristic feature of lanthanide single ion magnets (SIMs). 


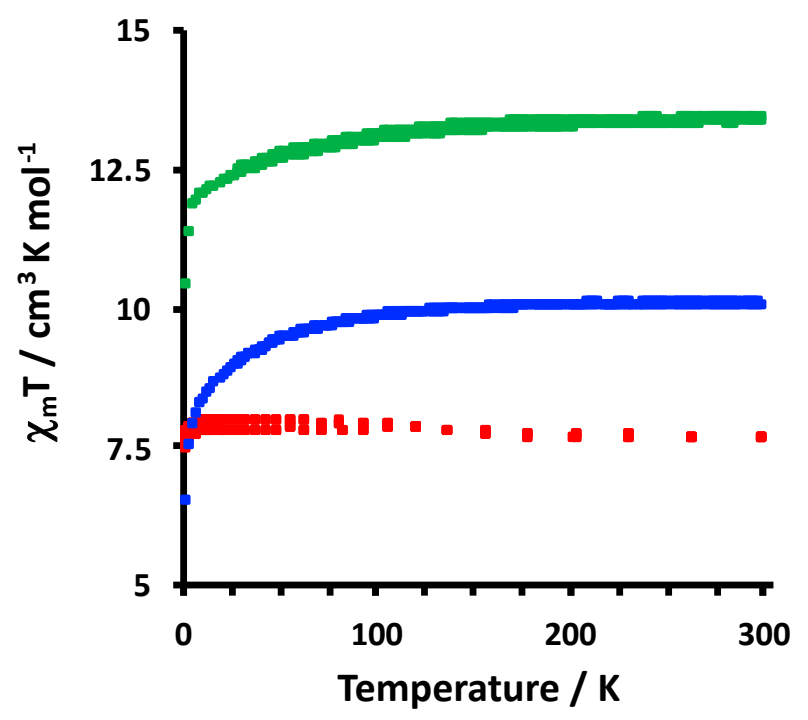

Figure 6. Variable temperature magnetic susceptibility data (ZFC/FC) for $\mathbf{1}$ (red), $\mathbf{2}$ (green), and 3 (blue) (external field 2000 Oe).

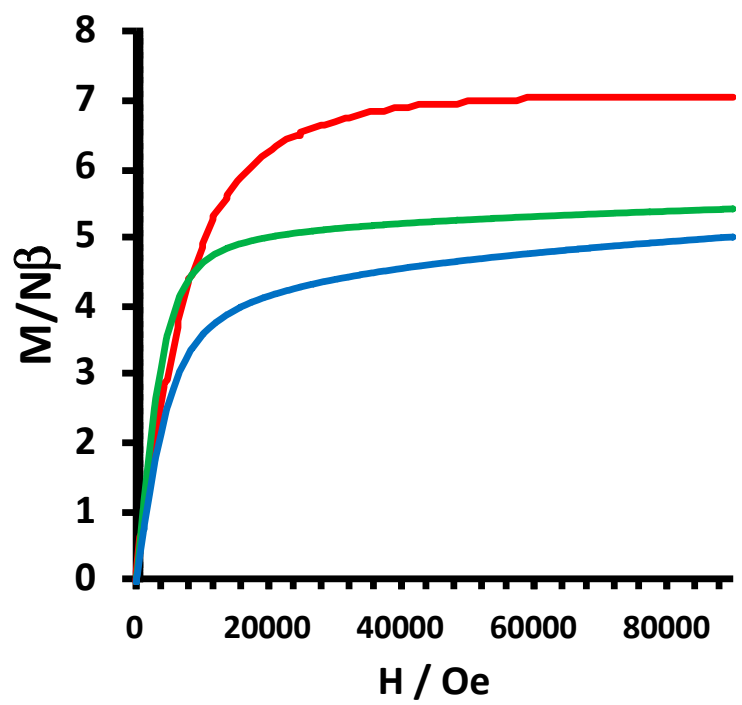

Figure 7. Magnetization versus field (0-9000 Oe) for $\mathbf{1}$ (red line), 2 (green line) and 3 (blue line) at $2 \mathrm{~K}$.

\subsection{Emission Spectroscopy of papl and 1-3}

Figure 8 shows the emission profiles of the papl ligand in $\mathrm{CH}_{2} \mathrm{Cl}_{2}$ at room temperature and at $77 \mathrm{~K}$. The emission spectra of the papl ligand obtained while screening different excitation wavelengths $\left(\lambda_{\text {exc }}=257\right.$ to $\left.600 \mathrm{~nm}\right)$ are presented in Figures S13-S15. Emission is not observed upon excitation of the free papl ligand in the lowest energy absorption band (Figure S14). This observation was previously reported for azo-type compounds and it was explained by the generation of a very polar excited state relaxing non-radiatively, associated with photochemical isomerization [19]. However, excitation at higher energy leads to dual emission at room temperature (Figure 8 (orange line) and Figures S13 and S14): a high energy band (325-425 nm) (with vibronic structure of similar energy $\left(\lambda_{\max }=365,384,419\right.$, and $\left.442 \mathrm{~nm}\right)$, and a lower energy band $(425-600 \mathrm{~nm})$ also featuring vibronic structure of similar energy $\left(\lambda_{\max }=490,517\right.$, and $\left.560 \mathrm{~nm}\right)$. At low temperature $(77 \mathrm{~K})$, the emission profile of the papl ligand consists of a broad band $(550 \mathrm{~nm}-750 \mathrm{~nm})$ without vibronic structure with $\lambda_{\max }=645 \mathrm{~nm}$. This very interesting and very complex emission profile of the free ligand results from a combination of multiple functionalities in its structure (e.g., azoaryl, phenanthrol and pyridyl 
moieties, including keto-enol forms), each of which may display a specific characteristic behavior upon excitation. Based on related systems [19], the high energy band (325-425 nm) is a locally excited (LE) state together with radiative relaxation from a mixing of higher $\mathrm{S}$ and $\mathrm{T}$. The emission of complex 1 confirms the triplet emission energy (see below). The lower energy band (425-600 $\mathrm{nm}$ ) is assigned to intramolecular change transfer states.

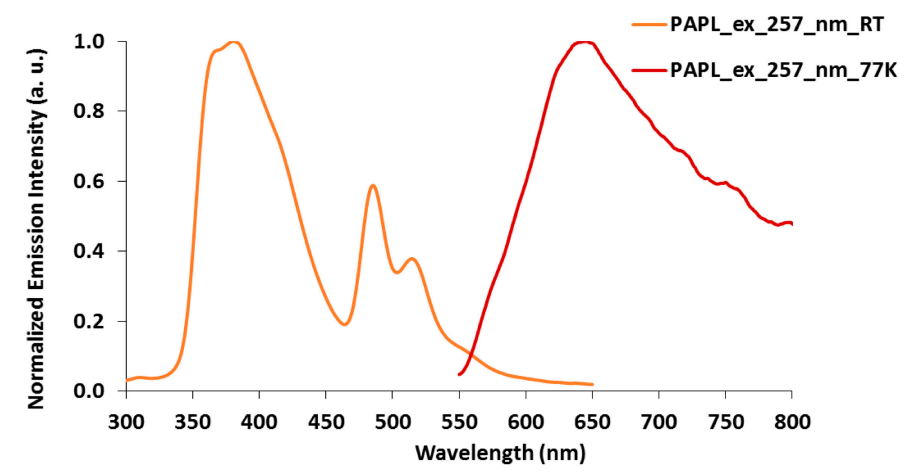

Figure 8. Emission profile of the papl ligand in $\mathrm{CH}_{2} \mathrm{Cl}_{2}$ at room temperature (orange line, $\lambda_{\max }=365$ (sh), 384,419 (sh), 490 (sh), 517, 544, and 560 (sh) nm; sh = shoulder) and at $77 \mathrm{~K}$ (red line, $\lambda_{\max }=645 \mathrm{~nm}$ ) $\left(\lambda_{\text {exc }}=257 \mathrm{~nm}\right.$; concentration $\left.1 \times 10^{-5} \mathrm{M}\right)$.

Figure 9 displays the emission profiles of 1 in $\mathrm{CH}_{2} \mathrm{Cl}_{2}$ at room temperature and at $77 \mathrm{~K}$. The emission spectra of 1 obtained while screening different excitation wavelengths $\left(\lambda_{\text {exc }}=257\right.$ to $\left.600 \mathrm{~nm}\right)$ are presented in Figure S16. The emission profiles for 2 and 3 are shown in Figure 10 and Figures S18-S26. In complexes of $\mathrm{Gd}(\mathrm{III})$, the high energy of the excited level of $\mathrm{Gd}(\mathrm{III})$ ion $\left({ }^{6} \mathrm{P}_{7 / 2}, 32,200 \mathrm{~cm}^{-1}\right)$ renders energy transfer from the ligand triplet state to the metal state impossible, while the heavy ion effect promotes the phosphorescence of the ligand. Therefore, these complexes are usually used to estimate the triplet energies of ligands [20]. Thus, the lowest triplet energy of the papl ligand $\left(17,000 \mathrm{~cm}^{-1}\right.$ or $590 \mathrm{~nm}$ ) was determined from the short-wavelength band edge of the phosphorescence spectrum of complex 1 at $77 \mathrm{~K}$. As is the case for the uncoordinated papl ligand, no emission is observed in complexes 1-3 when excited in the lowest energy absorption band (Figures S16, S18 and S19). When excited at higher energies, complexes 1-3 show only ligand-based emission at both room temperature and $77 \mathrm{~K}$ (Figures 9 and 10 and Figures S16, S18 and S19). The characteristic narrow-line emission from the excited levels of $\mathrm{Tb}(\mathrm{III})$ and $\mathrm{Dy}(\mathrm{III})$ ions [21] is not observed. The triplet energy of the papl ligand $\left(17,000 \mathrm{~cm}^{-1}\right)$ is lower than the excited levels of $\mathrm{Tb}$ (III) $\left({ }^{5} \mathrm{D}_{4}, 20,400 \mathrm{~cm}^{-1}\right)$ and Dy(III) $\left({ }^{4} \mathrm{~F}_{9 / 2}, 20,600 \mathrm{~cm}^{-1}\right)[20]$, therefore, these lanthanide ions are not sensitized by the papl ligand.

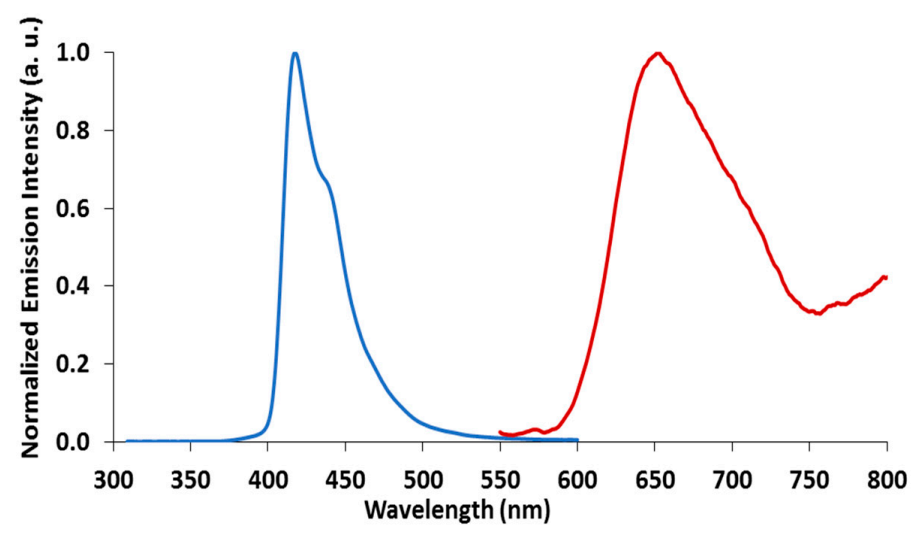

Figure 9. Emission profile of 1 in $\mathrm{CH}_{2} \mathrm{Cl}_{2}$ at room temperature (blue line, $\lambda_{\max }=419 \mathrm{~nm}$ ) and at $77 \mathrm{~K}$ (red line, $\left.\lambda_{\max }=652 \mathrm{~nm}\right)\left(\lambda_{\mathrm{exc}}=309 \mathrm{~nm}\right)\left(\right.$ concentration $\left.4 \times 10^{-5} \mathrm{M}\right)$. 


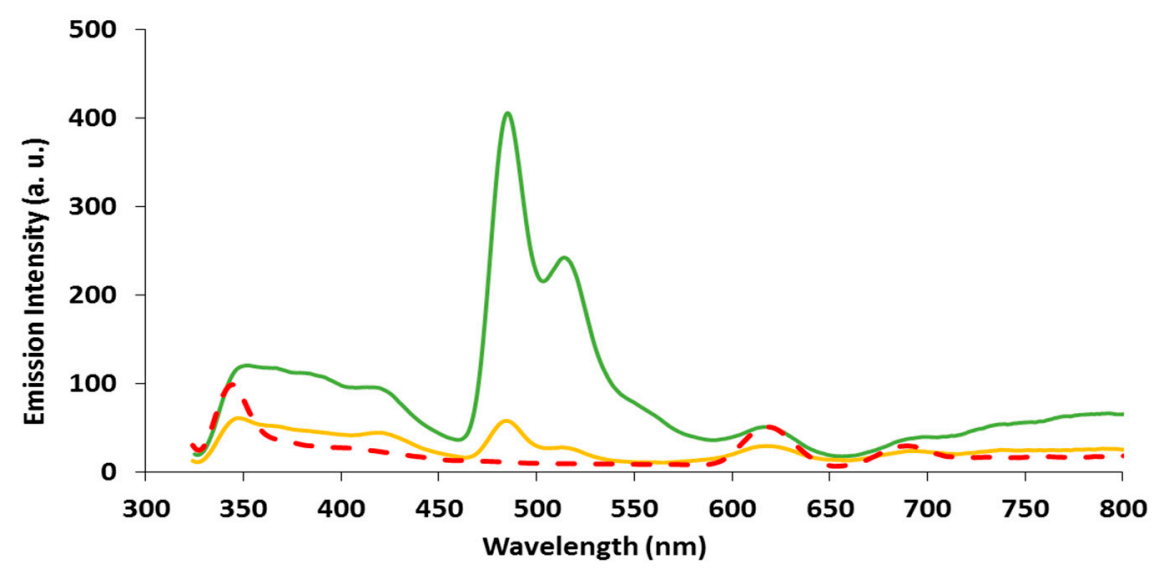

Figure 10. Emission profile of 2 (yellow line) and 3 (green line) in $\mathrm{CH}_{2} \mathrm{Cl}_{2}$ at $\mathrm{RT} ; \lambda_{\text {exc }}=309 \mathrm{~nm}$. Dashed red line is $\mathrm{CH}_{2} \mathrm{Cl}_{2}$.

Complex 1 in $\mathrm{CH}_{2} \mathrm{Cl}_{2}$ solution at room temperature (Figure 9, blue line) exhibits ligand-based emission at $419 \mathrm{~nm}$ when excited at higher energies ( $257 \mathrm{~nm}$ to $394 \mathrm{~nm}$ )-this state could be a higher triplet level of the ligand or a mixing between a higher singlet and a higher triplet (in the case of incomplete intersystem crossing at room temperature) or another type of excited state (e.g., intramolecular charge transfer) capable of relaxing radiatively. The energy of this level is estimated at $24,200 \mathrm{~cm}^{-1}$ (Figure S17). An enhancement of the emission intensity of this band with respect to the ligand is also observed for $\left[\mathrm{Gd}(\mathbf{p a p l})_{3}\right]$, as well as the disappearance of the emission band at $425-600 \mathrm{~nm}$. The $\mathrm{Tb}(\mathrm{III})$ and $\mathrm{Dy}(\mathrm{III})$ complexes exhibit very weak ligand-based emission: the high-energy band decreases with respect to the $\mathrm{Gd}(\mathrm{III})$ analog and the papl free ligand, while the low-energy band is the main feature of their emission spectra. However, the emission from this state(s) is partially quenched in the $\mathrm{Tb}$ (III) analog, and almost totally quenched in the Dy(III) analog, vs. the papl ligand and its Gd(III) complex. As mentioned before, the energy gap between the lowest energy excited (emissive) state of the metal ion and the ground state for $\mathrm{Gd}(\mathrm{III})$ ions $\left({ }^{6} \mathrm{P}_{7 / 2} \rightarrow{ }^{8} \mathrm{~S}_{7 / 2}\right)$ equals $32,200 \mathrm{~cm}^{-1}$, which is much higher than the energy of the excited state exhibiting emission at room temperature in $\mathbf{1}\left(24,200 \mathrm{~cm}^{-1}\right)$, thus explaining the absence of the quenching of the emission for this state [20]. In the case of $\mathrm{Dy}(\mathrm{III})$ and $\mathrm{Tb}$ (III) complexes $\mathbf{2}$ and 3, the quenching mechanism is not clear. The presence of $\mathrm{Dy}(\mathrm{III})$ or $\mathrm{Tb}(\mathrm{III})$ ions could contribute to the quenching of the ligand-based emission through the enhancement of inter-system crossing (ISC) to the lowest triplet state of the papl ligand, as expected based on the heavy ion effect. This latter state is thermally deactivated at room temperature, presumably by vibrational quenching, but it is emissive at $77 \mathrm{~K}$, as proven by the emission profile of the Gd(III) complex (Figure 9 and Figure S26). The absence of the same type of emission profile for $\mathrm{Dy}(\mathrm{III})$ and $\mathrm{Tb}(\mathrm{III})$ complexes at $77 \mathrm{~K}$ strongly suggests that other quenching pathway(s) are also involved in 2 and $\mathbf{3}$ (Figure S26). Considering the excitation at $309 \mathrm{~nm}\left(32,360 \mathrm{~cm}^{-1}\right.$, $4.0 \mathrm{eV}$ ), energy transfer could take place from ligand-based higher excited states (e.g., the state(s) at $325-425 \mathrm{~nm}$ and $425-600 \mathrm{~nm}$ ) to suitably positioned higher excited states of $\operatorname{Ln}$ (III) ions, followed exclusively by non-radiative relaxation (through the Ln(III) ion manifold or (back-)transfer to other non-emissive lower energy ligand-centered excited states). Bearing again in mind the excitation at $309 \mathrm{~nm}$, in case of 2, the possibility of Dy(III/II) reduction by a ligand excited state with generation of a non-radiatively decaying LMCT state could also be considered [20,22]. However, even though very interesting from the fundamental science perspective, identifying the exact nature of the ligand-based emission quenching pathways in $\mathbf{2}$ and $\mathbf{3}$ (especially considering that emission from the Dy(III) and $\mathrm{Tb}$ (III) ions was not identified below $800 \mathrm{~nm}$ ) would require more in-depth spectroscopic studies and it is beyond the scope of the present work. The effect of concentration of the sample on the emission intensity was also tested for complexes $\mathbf{2}$ and $\mathbf{3}$ reported in this work. The increase in complex concentration $\left(10^{-6}\right.$ to $\left.10^{-4} \mathrm{M}\right)$ results in the quenching of the emission at $425-600 \mathrm{~nm}$ in $\mathrm{CH}_{2} \mathrm{Cl}_{2}$ 
solution at both room temperature, and $77 \mathrm{~K}$ (Figures S24 and S25). In addition, no emission is detected in the solid state at room temperature. For both 2 and 3, a large emission band centered at 600-650 nm is observed, at high concentration, indicating that aggregation phenomena are (at least) partially implicated in generating the respective emissive state.

\section{Materials and Methods}

\subsection{General Procedures}

All reagents were commercially available and used as received. Ligands papl and qapl were prepared according to the literature procedures [10,11,15]. Anhydrous solvents were obtained from a Puresolve MD-4 solvent purification system. The electronic spectra were recorded in $\mathrm{CH}_{2} \mathrm{Cl}_{2}$ solutions on a Cary UV-Vis-NIR spectrophotometer 6000i (Agilent Technologies, Santa Clara, CA, USA) (concentration range of $10^{-4}-10^{-6} \mathrm{M}$ ). Luminescence spectra were obtained using a Perkin Elmer LS55 Luminescence Spectrometer (PerkinElmer, Waltham, MA, USA) equipped with a low temperature accessory. The measurements were performed in $\mathrm{CH}_{2} \mathrm{Cl}_{2}$ solutions (concentration range $10^{-4}-10^{-6} \mathrm{M}$ ), at room temperature, and at liquid nitrogen temperature $(77 \mathrm{~K})$. Wavelength accuracy is $\pm 1.0 \mathrm{~nm}$. FT-IR spectra were recorded on a Shimadzu IRAffinity spectrometer (Shimadzu America Inc., Columbia, MD, USA) as KBr discs. ESI mass spectra were obtained on a Bruker HCT Plus Proteineer LC-MS (Bruker Ltd., Milton, ON, Canada) with electrospray and a syringe pump was used for direct sample infusion. Elemental analyses were carried out by Canadian Microanalytical Services, LTD., Delta, BC, Canada.

CCDC 1844759 contains the supplementary crystallographic data for this paper. These data can be obtained free of charge via http:/ / www.ccdc.cam.ac.uk/conts/retrieving.html (or from the CCDC, 12 Union Road, Cambridge CB2 1EZ, UK; Fax: +44 1223 336033; E-mail: deposit@ccdc.cam.ac.uk)

\subsection{Electrochemical Measurements}

Cyclic voltammetry (CV) and differential pulse voltammetry (DPV) experiments were performed with a Bioanalytical Systems Inc. Epsilon electrochemical workstation. Compounds were dissolved in anhydrous solvent $\left(\mathrm{CH}_{2} \mathrm{Cl}_{2}\right)$, and then deoxygenated by sparging with $\mathrm{N}_{2}$ gas for 20 min. Solution concentrations were approximately $10^{-3} \mathrm{M}$ in analyte containing $0.5 \mathrm{M}$ supporting electrolyte $\left(\mathrm{Bu}_{4} \mathrm{NPF}_{6}\right)$. A three-electrode set-up was used including a glassy carbon working electrode, $\mathrm{Ag} / \mathrm{AgCl}$ reference electrode, and a platinum wire auxiliary electrode. The scan rate for $\mathrm{CV}$ experiments was $200 \mathrm{mV} / \mathrm{s}$. Parameters for the DPV experiments included a pulse amplitude of $50 \mathrm{mV}$, pulse width of $50 \mathrm{~ms}$, and pulse period of $100 \mathrm{~ms}$; scan rates were $40 \mathrm{mV} / \mathrm{s}$.

\subsection{Variable Temperature Magnetic Susceptibility Measurements}

DC Magnetic susceptibility and magnetization measurements for complex $\mathbf{1}$ were made with a 9 T DynaCool Physical Property Measurement System (Quantum Design) using the vibrating sample magnetometer option. The magnetic properties of complexes 2 and 3 were studied with a Quantum Design Magnetic Property Measurement System using a Superconducting Quantum Interference Device (SQUID). All samples were individually pelleted, weighed, tightly packed into plastic capsules and loaded into brass holders. The signal from an empty capsule and the brass holder were measured prior to filling and were found to be at least three orders of magnitude lower than the signal from the samples.

\subsection{Preparation of Complexes}

Gd(papl) 3 (1). 1-(2-pyridylazo)-2-phenanthrol (papl) (0.117 g, $0.391 \mathrm{mmol})$ was dissolved in chloroform $(5 \mathrm{~mL})$ and 5 drops of triethylamine were added to the solution. A solution of $\mathrm{Gd}\left(\mathrm{NO}_{3}\right)_{3} \cdot 6 \mathrm{H}_{2} \mathrm{O}(0.061 \mathrm{~g}, 0.135 \mathrm{mmol})$ in methanol $(5 \mathrm{~mL})$ was added quickly drop-wise to the ligand solution and then allowed to stir for $2 \mathrm{~h}$. A purple precipitate was produced, which was collected by 
vacuum filtration, washed with water, methanol and dried. Yield (0.088 g, 64\%). MS (ESI+): 1053.2 $\left(\mathrm{MH}^{+}\right)$. Anal. Calc'd for (\%) $\mathrm{C}_{57} \mathrm{H}_{36} \mathrm{~N}_{9} \mathrm{O}_{3} \mathrm{Gd}$ (found\%): C, 65.06 (65.34); H, 3.45 (3.39); N, 11.98 (11.87). FT-IR (KBr, cm $\left.{ }^{-1}\right): 3063$ (w), 1597 (m), 1584 (m), 1559 (m) 1510 (s) 1493 (s), 1437 (m), 1389 (w), 1319 (s), 1291 (m), 1275 (s), 1225 (s), 1208 (m), $1177(\mathrm{~m}), 1161$ (m), 1140 (s), $1101(\mathrm{~m}), 1034(\mathrm{~m}), 999$ (m), 928 (w), $754(\mathrm{~m}), 725(\mathrm{~m}), 637(\mathrm{w}), 517(\mathrm{w})$.

Dy(papl) $)_{3}$ (2). Papl (0.122 g, $\left.0.407 \mathrm{mmol}\right)$ was dissolved in chloroform (5 mL) and 5 drops of triethylamine were added to the solution. A solution of $\mathrm{Dy}\left(\mathrm{NO}_{3}\right)_{3} \cdot \mathrm{xH}_{2} \mathrm{O}(0.051 \mathrm{~g})$ in methanol ( $5 \mathrm{~mL}$ was added quickly drop-wise to the ligand solution and stirred for $2 \mathrm{~h}$. A purple precipitate was produced that was collected by vacuum filtration, washed with water, methanol and dried. Yield (0.086 g, 59\%). MS (ESI+): $1059.2\left(\mathrm{MH}^{+}\right)$. Anal. Calc'd for (\%) $\mathrm{C}_{57} \mathrm{H}_{36} \mathrm{~N}_{9} \mathrm{O}_{3} \mathrm{Dy}$ (found\%): C, 64.74 (65.19); H, 3.43 (3.25); N, 11.92 (12.00). FT-IR (KBr, cm $\left.{ }^{-1}\right)$ : 3062 (w), 1597 (m), 1584 (m), 1559 (m), 1512 (s), 1493 (s), 1437 (m), 1389 (w), 1341 (m), 1319 (s), 1294 (m), 1277 (s), 1227 (s), 1207 (m), 1179 (s), $1161(\mathrm{~m}), 1140(\mathrm{~s}), 1126(\mathrm{~m}), 1101(\mathrm{~m}), 1034(\mathrm{~m}) 999(\mathrm{~m}), 928(\mathrm{w}), 872(\mathrm{w}), 754(\mathrm{~m}), 725(\mathrm{~m}), 654(\mathrm{w})$.

$\mathbf{T b}(\text { papl })_{3} \cdot \mathbf{0 . 4} \mathrm{CHCl}_{3}$ (3). Papl $(0.10 \mathrm{~g}, 0.33 \mathrm{mmol})$ was dissolved in chloroform $(5 \mathrm{~mL})$ and 5 drops of triethylamine were added to the solution. A solution of $\mathrm{Tb}\left(\mathrm{NO}_{3}\right)_{3} \cdot 5 \mathrm{H}_{2} \mathrm{O}(0.049 \mathrm{~g}, 0.113 \mathrm{mmol})$ in methanol $(5 \mathrm{~mL})$ was added drop-wise to the ligand solution and allowed to stir for $2 \mathrm{~h}$. A purple precipitate was produced, which was collected by vacuum filtration, washed with water, methanol and dried. Yield (0.074 g, 62\%). MS (ESI+): $1054.2\left(\mathrm{MH}^{+}\right)$. Anal. Calc'd for $(\%) \mathrm{C}_{57} \mathrm{H}_{36} \mathrm{~N}_{9} \mathrm{O}_{3} \mathrm{~Tb} \cdot 0.4 \mathrm{CHCl}_{3}$ (found\%): C, 62.58 (62.60); H, 3.33 (3.21); N, 11.44 (11.50). FT-IR (KBr, cm $\left.{ }^{-1}\right)$ : 3065 (w), 1597 (m), $1584(\mathrm{~m}), 1559(\mathrm{~m}), 1510(\mathrm{~m}), 1491(\mathrm{~m}), 1437(\mathrm{~m}), 1387(\mathrm{w}), 1341(\mathrm{~m}), 1319(\mathrm{~s}), 1292(\mathrm{~m}), 1277$ (s), 1227 (s), $1207(\mathrm{~m}), 1179(\mathrm{~m}), 1161(\mathrm{~m}), 1140(\mathrm{~s}), 1101(\mathrm{~m}), 1034(\mathrm{w}), 999(\mathrm{~m}), 871(\mathrm{w}), 756(\mathrm{~m}), 725(\mathrm{~m})$.

Ho(qapl) $)_{3} \cdot \mathbf{1 . 1} \mathbf{C H}_{2} \mathbf{C l}_{\mathbf{2}}$ (4). Qapl $(0.075 \mathrm{~g}, 0.23 \mathrm{mmol})$ was dissolved in chloroform $(15 \mathrm{~mL})$ and 10 drops of triethylamine were added to the solution. A solution of $\mathrm{HoCl}_{3} \cdot 6 \mathrm{H}_{2} \mathrm{O}(0.029 \mathrm{~g}, 0.077 \mathrm{mmol})$ in methanol $(15 \mathrm{~mL})$ was added drop-wise to the ligand solution and allowed to stir for $12 \mathrm{~h}$ at approximately $35^{\circ} \mathrm{C}$. A red-purple precipitate was produced, which was collected by vacuum filtration, washed with water, methanol and dried. Yield $(0.068 \mathrm{~g}, 75 \%)$. Single crystals of 4 were grown by slow evaporation of DCM/ $\mathrm{CH}_{3} \mathrm{CN}$ solutions (approximately $2 \mathrm{mg}$ in $11 \mathrm{~mL}$ DCM and $2 \mathrm{~mL} \mathrm{CH} 3 \mathrm{CN}$ ). MS (ESI+): $1210.2\left(\mathrm{MH}^{+}\right)$. Anal. Calc'd for (\%) $\mathrm{C}_{69} \mathrm{H}_{42} \mathrm{~N}_{9} \mathrm{O}_{3} \mathrm{Ho} \cdot 1.1 \mathrm{CH}_{2} \mathrm{Cl}_{2}$ (found\%): C, 64.59 (64.68); H, 3.42 (3.59); N, 9.67 (9.49). FT-IR (KBr, cm $\left.{ }^{-1}\right)$ : $3447(\mathrm{~m}), 3061(\mathrm{w}), 3019$ (w), 1605 (m), $1582(\mathrm{~m})$, $1558(\mathrm{~m}), 1510(\mathrm{~m}), 1487(\mathrm{~m}), 1379(\mathrm{~m}), 1341$ (s), 1325 (s), 1300 (s), 1222 (s), $1204(\mathrm{~m}), 1155$ (w), 1105 (w), $1087(w), 1051(w), 1037(w), 968(w), 874(w), 831(w), 810(w), 785(w), 752(m), 727(m), 692(w)$. $\lambda_{\max }(\mathrm{THF})=506 \mathrm{~nm}$.

\section{Conclusions}

Herein, we have reported the first lanthanide ion complexes 1-3 with the arylazo ligand papl. In these complexes, three equivalents of the deprotonated ligand bind to the metal ion to produce nine coordinate species with a likely tricapped trigonal prismatic geometry at the lanthanide ion, analogous to the $\mathrm{Ho}(\mathbf{q a p l})_{3}$ complex also reported in this work. As anticipated, complexes 1-3 exhibit similar spectroscopic and electrochemical features to transition metal complexes containing papl, including intense visible absorption bands and rich cathodic electrochemical behavior. Of note, we investigated the luminescent properties of 1-3 and the uncoordinated papl ligand since there are very few reports of the emission properties of lanthanide complexes coordinated to arylazo ligands. In all cases, the typical sharp lanthanide ion centered luminescence is not observed and the emission profiles of 1-3 all resembled that obtained from the uncoordinated papl ligand. The emission intensities are dependent on the ion with $\mathrm{Gd}$ the most intense and very weak emission observed from the $\mathrm{Tb} / \mathrm{Dy}$ complexes. While no slow relaxation of the magnetization was observed for the $\mathrm{Tb}$ or Dy complexes described in this work, we are pursuing other heteroleptic lanthanide complexes containing papl using $\left[\mathrm{Ln}(\mathbf{t t a})_{3}\right]\left(\mathbf{t t a}^{-}=\right.$2-thenoyltrifluoroacetonate) or $\left[\mathrm{Ln}(\mathbf{h f a c})_{3}\right]\left(\mathrm{hfac}^{-}=\right.$hexafluoroacethylacetonate) salts, which have been shown to produce multifunctional luminescent SIMs with other redox active ligand types [23]. 
Supplementary Materials: Supplementary Materials can be found at http:/ / www.mdpi.com/2304-6740/6/2/ 56/s1. Figures S1-S26: ESI, CIF, electrochemical, variable temperature magnetic susceptibility and luminescence data for 1-3. Table S1: Crystal data and structure refinement for 4, Cif and checkcif file of 4.

Author Contributions: M.T.L. conceived and designed the experiments; R.A.T. synthesized and characterized the papl ligand and complexes 1-3; M.C. and J.Y. performed the photophysical experiments; N.M.B. prepared qapl, complex 4 and grew single crystals; A.J.L. solved the crystal structure of 4; C.R.W., H.J.S. and C.M. designed and completed the variable temperature magnetic susceptibility experiments; and M.T.L. wrote the paper with contributions from all co-authors.

Acknowledgments: M.T.L. acknowledges funding from NSERC, CFI, Canada Research Chairs Program, Brandon and Brock University and the University of Manitoba for providing an adjunct position. M.C. thanks NSERC and the FRQNT and Université de Montréal for graduate fellowships. Y.J. thanks MITACS for a student fellowship. C.R.W. acknowledges funding from NSERC, CFI, the Canada Research Chairs Program (Tier II), CIFAR, and the University of Manitoba for providing an adjunct position.

Conflicts of Interest: The authors declare no conflict of interest.

\section{References}

1. Bottrill, M.; Kwok, L.; Long, N.J. Lanthanides in magnetic resonance imaging. Chem. Soc. Rev. 2006, 35, 557-571. [CrossRef] [PubMed]

2. Harvey, P.; Kuprov, I.; Parker, D. Lanthanide complexes as paramagnetic probes for 19F magnetic resonance. Eur. J. Inorg. Chem. 2012, 2015-2022. [CrossRef]

3. Tsitovich, P.B.; Burns, P.J.; McKay, A.M.; Morrow, J.R. Redox-activated MRI contrast agents based on lanthanide and transition metal ions. J. Inorg. Biochem. 2014, 133, 143-154. [CrossRef] [PubMed]

4. Zhang, M.; Qu, Z.B.; Han, C.M.; Lu, L.F.; Li, Y.Y.; Zhou, T.; Shi, G. Time-resolved probes and oxidase-based biosensors using terbium(III)-guanosine monophosphate-mercury(II) coordination polymer nanoparticles. Chem. Commun. 2014, 50, 12855-12858. [CrossRef] [PubMed]

5. Bünzli, J.-C.G. Lanthanide Luminescence for Biomedical Analyses and Imaging. Chem. Rev. 2010, 110, 2729-2755. [CrossRef] [PubMed]

6. Woodruff, D.N.; Winpenny, R.E.P.; Layfield, R.A. Lanthanide single-molecule magnets. Chem. Rev. 2013, 113, 5110-5148. [CrossRef] [PubMed]

7. Sorace, L.; Benelli, C.; Gatteschi, D. Lanthanides in molecular magnetism: Old tools in a new field. Chem. Soc. Rev. 2011, 40, 3092-3104. [CrossRef] [PubMed]

8. Rinehart, J.; Long, J. Exploiting single-ion anisotropy in the design of f-element single-molecule magnets. Chem. Sci. 2011, 2, 2078-2085. [CrossRef]

9. Samanta, S.; Ghosh, P.; Goswami, S. Recent advances on the chemistry of transition metal complexes of 2-(arylazo)pyridines and its arylamino derivatives. Dalton Trans. 2012, 41, 2213-2226. [CrossRef] [PubMed]

10. Chiswell, B.; Lions, F.; Tomlinson, M.L. Tridentate Chelate Compounds. IV. Metal Complexes from a-Diketone Mono-a-pyridylhydrazone Type Ligands. Inorg. Chem. 1961, 611, 322-327. [CrossRef]

11. Van Damme, N.; Lough, A.J.; Gorelsky, S.I.; Lemaire, M.T. Molecular and electronic structures of complexes containing 1-(2-pyridylazo)-2-phenanthrol (PAPL): Revisiting a redox-active ligand. Inorg. Chem. 2013, 52, 13021-13028. [CrossRef] [PubMed]

12. Taylor, R.A.; Lough, A.J.; Lemaire, M.T. Spin-crossover in a homoleptic cobalt(II) complex containing a redox-active NNO ligand. J. Mater. Chem. C 2016, 4, 455-459. [CrossRef]

13. Takahashi, K.; Kawamukai, K.; Okai, M.; Mochida, T.; Sakurai, T.; Ohta, H.; Yamamoto, T.; Einaga, Y.; Shiota, Y.; Yoshizawa, K. A New Family of Anionic FeIII Spin Crossover Complexes Featuring a Weak-Field $\mathrm{N}_{2} \mathrm{O}_{4}$ Coordination Octahedron. Chem. A Eur. J. 2016, 22, 1253-1257. [CrossRef] [PubMed]

14. Andreiadis, E.S.; Imbert, D.; Pécaut, J.; Demadrille, R.; Mazzanti, M. Self-assembly of highly luminescent lanthanide complexes promoted by pyridine-tetrazolate ligands. Dalton Trans. 2012, 41, 1268. [CrossRef] [PubMed]

15. Taylor, R.A.; Bonanno, N.M.; Mirza, D.; Lough, A.J.; Lemaire, M.T. A trivalent cobalt complex with the new redox-active ligand 10-(8-quinolylazo)-9-phenanthrol (qapl). Polyhedron 2017, 131, 34-39. [CrossRef]

16. Bonanno, N.M.; van Damme, N.; Lough, A.J.; Lemaire, M.T. Transition metal complexes containing a ditopic redox active ligand featuring very intense visible absorption bands. Dyes Pigment. 2015, 123, 212-217. [CrossRef] 
17. Zhou, J.L.; Xu, Y.H.; Jin, X.X.; Xiao, S.; Yi, H.B.; Yan, J. Synthesis and characterization of luminescent zinc complexes containing redox-active 1-(2-pyridylazo)-2-acenaphthequinol ligands with nonlinear optical property. Inorg. Chem. Commun. 2016, 64, 67-70. [CrossRef]

18. Wu, Y.; Wang, J.; Kong, X.; Sheng, C.; Wang, R.; Peyghambarian, N.; Norwood, R.A.; Zheng, Z. A dinuclear europium(III) complex with thenoyltrifluoroacetonato and 1-(2-pyridylzao)-2-naphtholato ligands and its optical properties. Inorg. Chim. Acta 2011, 370, 346-352. [CrossRef]

19. Santos, F.D.S.; Descalzo, R.R.; Gonçalves, P.F.B.; Benvenutti, E.V.; Rodembusch, F.S. Evidence for excited state intramolecular charge transfer in benzazole-based pseudo-stilbenes. Phys. Chem. Chem. Phys. 2012, 14, 10994-11001. [CrossRef] [PubMed]

20. Shuvaev, S.; Utochnikova, V.; Marciniak, Ł.; Freidzon, A.; Sinev, I.; Van Deun, R.; Freire, R.O.; Zubavichus, Y.; Grünert, W.; Kuzmina, N. Lanthanide complexes with aromatic o-phosphorylated ligands: Synthesis, structure elucidation and photophysical properties. Dalton Trans. 2014, 43, 3121-3136. [CrossRef] [PubMed]

21. Bünzli, J.-C.G.; Piguet, C. Taking advantage of luminescent lanthanide ions. Chem. Soc. Rev. 2005, 34, 1048-1077. [CrossRef] [PubMed]

22. Meyer, A.U.; Slanina, T.; Heckel, A.; König, B. Lanthanide Ions Coupled with Photoinduced Electron Transfer Generate Strong Reduction Potentials from Visible Light. Chem. Eur. J. 2017, 23, 7900-7904. [CrossRef] [PubMed]

23. Pointillart, F.; le Guennic, B.; Cador, O.; Maury, O.; Ouahab, L. Lanthanide Ion and Tetrathiafulvalene-Based Ligand as a "magic" Couple toward Luminescence, Single Molecule Magnets, and Magnetostructural Correlations. Acc. Chem. Res. 2015, 48, 2834-2842. [CrossRef] [PubMed]

(C) 2018 by the authors. Licensee MDPI, Basel, Switzerland. This article is an open access article distributed under the terms and conditions of the Creative Commons Attribution (CC BY) license (http:/ / creativecommons.org/licenses/by/4.0/). 